\title{
Erratum to: Reevaluation of the hadronic contributions to the muon $g-2$ and to $\alpha\left(M_{Z}^{2}\right)$
}

\author{
M. Davier ${ }^{1}$, A. Hoecker ${ }^{2, a}$, B. Malaescu ${ }^{1, b}$, Z. Zhang $^{1}$ \\ ${ }^{1}$ Laboratoire de l'Accélérateur Linéaire, IN2P3/CNRS, Université Paris-Sud 11, Orsay, France \\ ${ }^{2}$ CERN, 1211 Geneva 23, Switzerland
}

Received: 2 January 2012 / Published online: 9 February 2012

(C) The Author(s) 2012. This article is published with open access at Springerlink.com

\section{Erratum to: Eur. Phys. J. C (2011) 71:1515 DOI 10.1140/epjc/s10052-010-1515-z}

Our above-mentioned publication [Eur. Phys. J. C 71:1515 (2011)] contains a mistake in the central value of the $K \bar{K} \pi$ contribution (set accidentally to zero) to the running of the electromagnetic coupling constant at the $Z$-boson mass, $\alpha\left(M_{Z}^{2}\right)$, which affects the final evaluation of this quantity. With the corrected $\alpha\left(M_{Z}^{2}\right)$, the shift in the Higgs mass obtained from the global electroweak fit amounts to $+7 \mathrm{GeV}$ (instead of the $+12 \mathrm{GeV}$ given in our publication) with respect to previous evaluations of $\alpha\left(M_{Z}^{2}\right)$.

The following items in Eur. Phys. J. C 71:1515 (2011) should be corrected.

- The last two sentences of the abstract should read: For the $e^{+} e^{-}$-based five-quark hadronic contribution to $\alpha\left(M_{Z}^{2}\right)$ we find $\Delta \alpha_{\text {had }}^{(5)}\left(M_{Z}^{2}\right)=(275.7 \pm 1.0) \cdot 10^{-4}$. The reduced electromagnetic coupling strength at $M_{Z}$ leads to an increase by $7 \mathrm{GeV}$ in the central value of the Higgs boson mass obtained by the standard Gfitter fit to electroweak precision data.

- In Table 2, the line giving the " $K \bar{K} \pi$ (partly from isospin)" contribution should read $0.76 \pm 0.02 \pm 0.04 \pm$ 0.02 in the rightmost column $\left(\Delta \alpha_{\mathrm{had}}\left(M_{Z}^{2}\right)\right)$. The corresponding "Sum" contribution (last line) should read $274.97 \pm 0.17 \pm 0.78 \pm 0.37 \pm 0.18_{\psi} \pm 0.52_{\mathrm{QCD}}$.

The online version of the original article can be found under doi:10.1140/epjc/s10052-010-1515-z.

a e-mail: Andreas.Hoecker@cern.ch

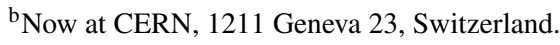

- Equation (24) should read:

$$
\Delta \alpha_{\text {had }}\left(M_{Z}^{2}\right)=(275.0 \pm 1.0) \cdot 10^{-4},
$$

- In the paragraph after (24) it should read: The corresponding $\tau$-based result reads $\Delta \alpha_{\text {had }}\left(M_{Z}^{2}\right)=(276.1 \pm$ $1.1) \cdot 10^{-4}$.

- Equation (25) should read

$\alpha^{-1}\left(M_{Z}^{2}\right)=128.952 \pm 0.014$.

- Figure 10 and caption should appear as

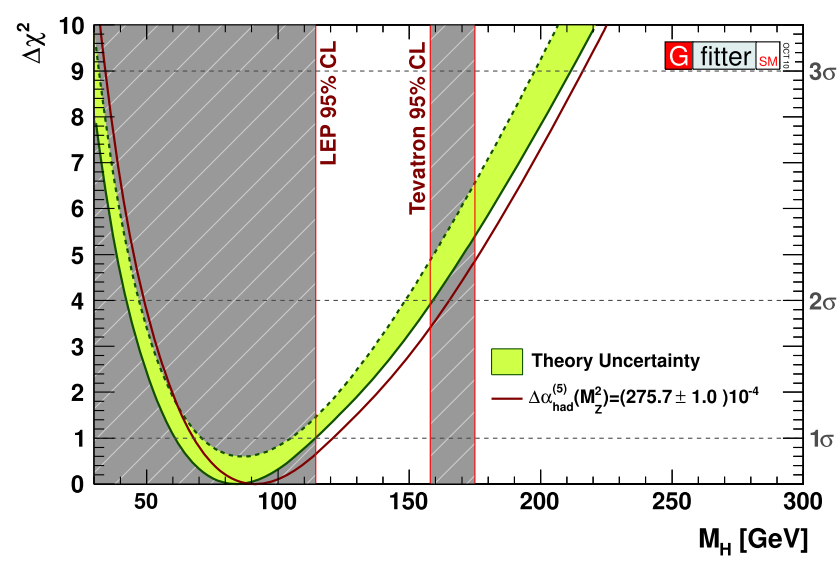

Fig. 10 Standard Gfitter electroweak fit result [55] (green shaded band) and the result obtained for the new evaluation of $\Delta \alpha_{\text {had }}\left(M_{Z}^{2}\right)$ (red solid curve). The legend displays the corresponding five-quark contribution, $\Delta \alpha_{\text {had }}^{(5)}\left(M_{Z}^{2}\right)$, where the top term of $-0.72 \cdot 10^{-4}$ is excluded. A shift of $+7 \mathrm{GeV}$ in the central value of the Higgs boson is observed

- In the paragraph after (25) it should read: The fitted Higgs mass shifts from previously $84_{-23}^{+30} \mathrm{GeV}$ to $91_{-23}^{+30} \mathrm{GeV}$. The stationary error of the latter value, in 
spite of the improved accuracy in $\Delta \alpha_{\text {had }}\left(M_{Z}^{2}\right)$, is due to the logarithmic $M_{H}$ dependence of the fit observables. The new $95 \%$ and $99 \%$ upper limits on $M_{H}$ are $163 \mathrm{GeV}$ and $193 \mathrm{GeV}$, respectively.

- In the "Conclusions" section it should read: ... whileon the other hand-relaxes the tension between the direct Higgs searches and the electroweak fit by $7 \mathrm{GeV}$ for the Higgs mass.
Open Access This article is distributed under the terms of the Creative Commons Attribution License which permits any use, distribution, and reproduction in any medium, provided the original author(s) and the source are credited. 